\title{
Design Simplification by Analogical Reasoning
}

\author{
Marton E. Balazs, and David C. Brown \\ Richmond University, UK \\ Worcester Polytechnic Institute, USA
}

\begin{abstract}
This paper presents our research on design simplification by analogical reasoning. It defines a "simplification problem", then describes our approach to solving design simplification problems by analogical reasoning. One of the important contributions of the research is the use of relevance for guiding the analogical reasoning process. Its benefits are analyzed using a set of experiments. Finally, the paper draws conclusions and proposes directions for future research
\end{abstract}
Key words: Design complexity, design simplification, model-based reasoning, analogical reasoning

\section{INTRODUCTION}

Ever since artifacts have been produced, improving them has been a common human activity. Improving an artifact refers to modifying it so that it will be either easier to produce, or easier to use, or easier to fix, or easier to maintain, and so on. In all of these cases, "easier" means less resource is required for the process. A "resource" can ultimately be expressed by some measure of cost (such as time or money).

At the core of many improvements is the notion of reduction of complexity, i.e., simplification. For instance, the less complicated an artifact is, as measured by its number of parts, the easier it will be to manufacture. Clearly, the actual cost of the manufacturing process will depend on the technological sophistication of the manufacturer, the experience and skill of 
the workers, and so on. However, as opposed to cost, the complexity of an artifact gives an objective characterization of the manufacturing difficulty.

Studying simplification is a very important research direction since it targets the understanding and simulation of a basic human (cognitive) activity. This can lead to important theoretical and applied results. The study of simplification may provide a context for studying human creativity as a by-product of goal-directed reasoning processes.

This paper presents results of our research on performing simplification of designs by analogical reasoning (Balazs 1999). It defines design simplification; describes our approach to solving design simplification problems by using goal-driven analogical reasoning; describes the implementation; analyzes some experimental results; discusses related work, draws some conclusions and outlines plans for future research.

\section{SIMPLIFYING DESIGNS}

Simplifying a design means to reduce its complexity. We view complexity as a way of characterizing designs from a given point of view, expressed as context, aspect and measure. A context for characterizing a design's complexity refers to a process that can be performed on the design (e.g., describing it, producing it, using it, etc.). For a given context, an aspect is the collection of those elements of the design which play a role in their characterization in the context considered (e.g, its structure, its behavior or its function). Finally, for a given context and aspect, a measure is a function that assigns to a design a numeric value that characterizes the complexity of the design in the given context and aspect. For example, counting the components of a design can be a measure of complexity defined for the context of manufacturing, in the aspect of structure: it characterizes the number of components that have to be manufactured. When discussing the complexity of a design we will assume that a point of view has been chosen.

We say that a design $A$ is simpler than another design $B$ if the complexity of $A$ is lower than the complexity of $B$. In this case we also say that the designs $A$ and $B$ are in the simpler-then relation. We will call an instance of the simpler-than relation a simplification.

A simplification can be discovered: a) by measuring and then comparing the complexities of two given designs, or b) solving a design simplification problem. In our research we are interested in solving design simplification problems.

Given a design, and a point of view (context, aspect and measure) the design simplification problem is the problem of finding another design that has the same function as the original design and with complexity less than 
that of the original design. The process of solving a simplification problem is a simplification process (or just a simplification). The research question is "How can design simplification problems be solved in an effective and efficient way?".

Simplification can be done in different ways: one could apply simplification rules acquired from designers, or one could use previously performed simplifications as a model for simplifying a design. Reusing known simplifications by adapting them to new problems allows the solution of new simplification problems. If the reuse is based on generalization and mapping to a different domain, new and previously unsolved simplification problems can be solved. In addition, the generalization would support the search for general simplification principles. This is why we chose to use analogical reasoning to perform simplification. Our approach to use "goaldirected" analogical reasoning.

\subsection{A Design Simplification Example}

Consider the design of a personal fax machine: Figure la (Petroski 1996). The functions of this design are to allow the sending and receiving of faxes. The complexity of this design in the context of manufacturing, with respect to the aspect of structure as measured by the number of its components is 6 .

A solution to the simplification problem specified by this design and the point of view considered is presented in Figure $1 \mathrm{~b}$ (Petroski 1996). This new design has the same functions as the original one and a reduced complexity of 5 . We propose that the simplification process that produces the "New Fax" design from the "Old Fax" design consists of the following steps:

1. A database of known simplifications is searched for simplifications that could be used as model for solving the problem. This search is based on measuring the degree of similarity between the "Old Fax" and designs that are the "more complex" members of known simplifications. The measure must favor designs that have a structure (i.e., system of relations) similar to the "Old Fax". Note here that it is possible that individual simplifications may refer to only some parts of designs. We say that those parts are relevant to the corresponding simplification. This observation can be exploited in searching the database of known simplifications as well as in subsequent phases of the simplification process. If we use a database of simplifications that contains examples of simplification for arithmetic expressions the simplification shown in Figure 2 may be retrieved as the best model for solving the simplification problem at hand. The simplification retrieved is called the source analog. 
2. Next the simplification knowledge represented in the source analog has to be transferred to the problem domain and applied to the simplification problem. Both the transfer and the application may require adaptations such that the result is valid in the problem domain. For the example considered, the simplification process transferred from the source simplification can be described by:

REPLACE: a motor driving a roller and a motor driving a roller BY: $\quad$ a motor driving (a roller and $a$ roller)

Applying this process to the "Old Fax" results in the simpler "New Fax" (Figure 1b).

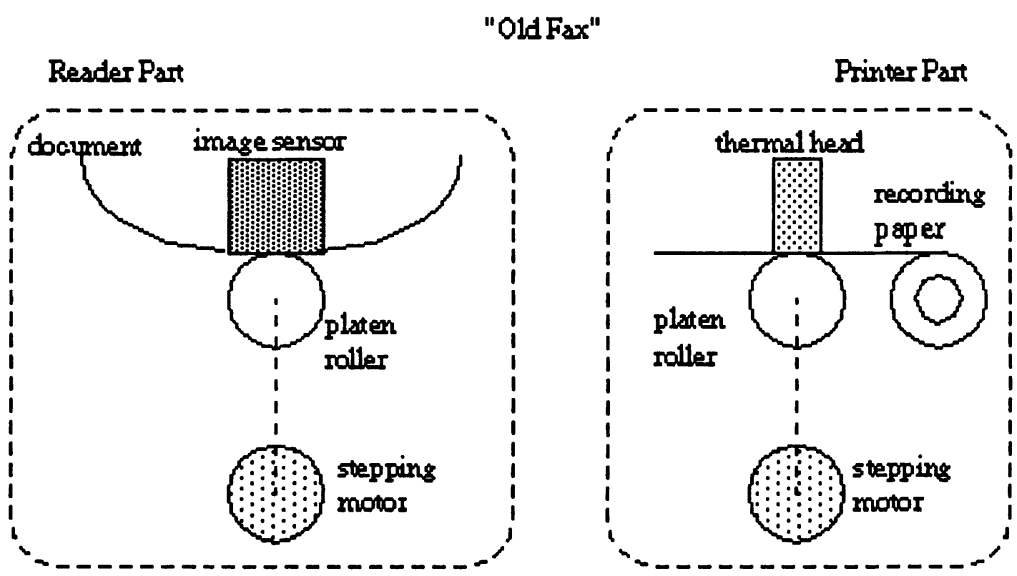

(a)

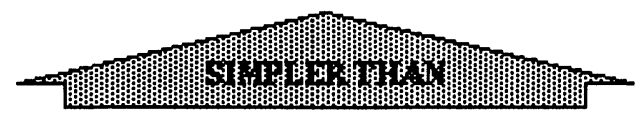

"New Fax"

Resder Part

Printer Part

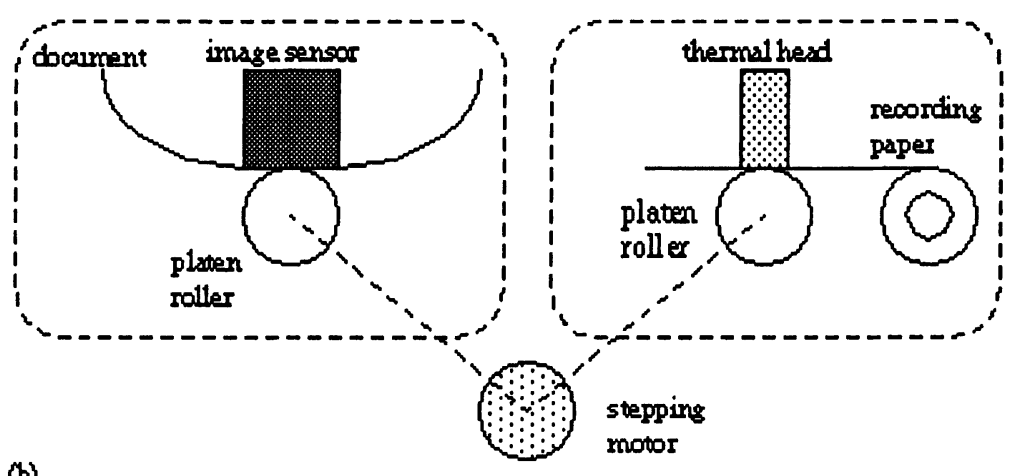

(b)

Figure 1. Simplification of a personal fax machine. Adapted from (Petroski 1996) 


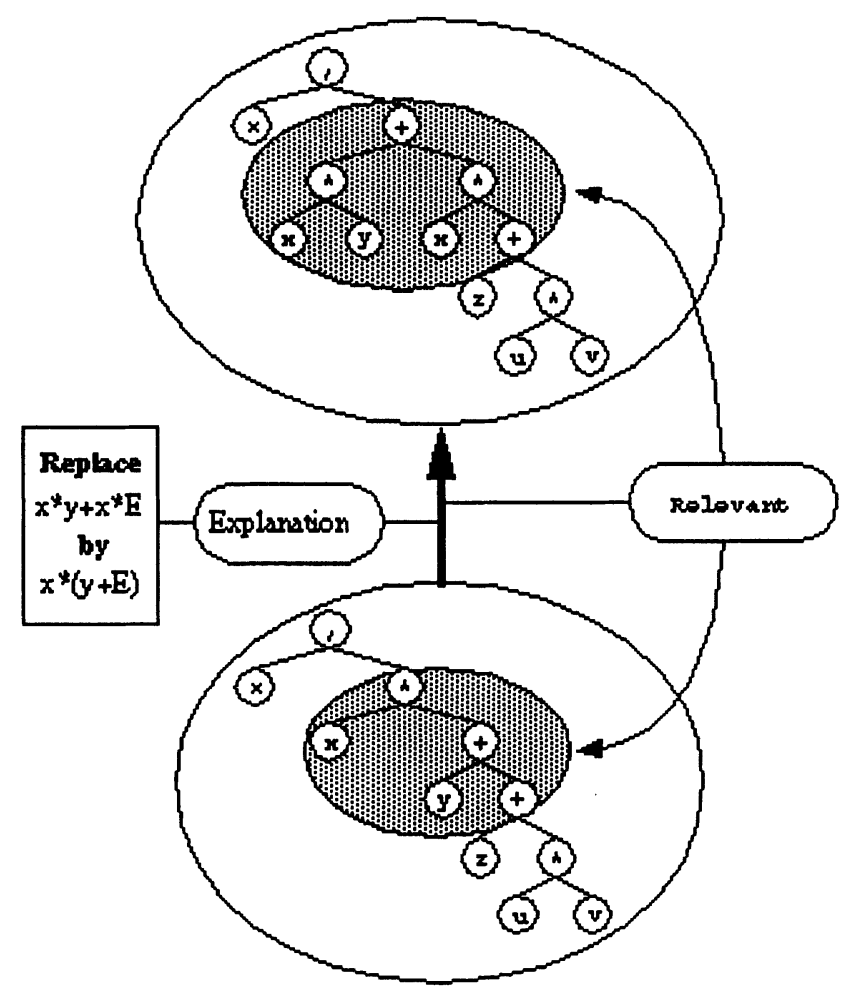

Figure 2. Source analog retrieved for the fax design simplification problem

The actual processing is much more complicated than described above. In order to be able to perform it the following questions must be answered (Bhatta and Goel 1994):

What should be the content and representation of source analogs? How is the target problem specified? Given a target problem, how might the retrieval of the source analog occur? Once a source analog has been retrieved, how can it be mapped onto the target problem and how will this mapping be used to transfer the problem solving knowledge? How can the solution to the target problem be completed? How will a solution to the target problem be evaluated? How can it be decided whether a useful generalization over the source problem and the target problem can be built. How can such a generalization be built? How can it be decided whether the target problem and its solution are different (novel) enough to be worth storing for later use? How can the generalization and/or the target problem be stored in the database of problems for later use? Our research proposes answers to all these questions for design simplification by analogical reasoning, taking into consideration the possibility of using the simplification goal to improve the performance of the processing. 


\section{DESIGN SIMPLIFICATION BY ANALOGICAL REASONING}

The model of the goal-driven analogical reasoning process we are proposing for solving design simplification problems was derived from a quite general model of analogical reasoning. The analogical reasoned process consists of the following phases:

Retrieval of candidate source analogs: this phase selects from the set of known simplifications those that have the same point of view as the problem, and which are "similar" to the problem. Similarity is measured in terms of the number and kind of elements (e.g., components, relations and attributes) they share.

Selection of the source analog: each candidate analog retrieved has associated with it a score which measures its similarity to the object to be simplified. This score is used to select the simplification closest to the problem.

Mapping of the source analog onto the problem: this phase will produce several "global mappings" that are consistent sets of correspondences between relevant elements in the source analog, and elements in the problem.

Selection of the best global mapping: each of the global mappings obtained will be evaluated for quality by combining the scores of the member correspondences (e.g., correspondences between relations will assigned higher scores than correspondences between attributes). The correspondence scores are assigned at the time of retrieval. The global mapping with the highest score will be used for transferring the simplification knowledge.

Transfer of simplification knowledge: the best global mapping will be used to produce several candidate simplifications by associating the unmapped elements in the source analog with elements in the problem.

Application and evaluation of candidate simplifications: all of the candidate simplifications are applied to the simplification problem, producing new objects. The objects produced will be evaluated against the problem constraints and for the simplification condition. An object produced is discarded if it doesn't satisfy the constraints or isn't simpler than the original.

Selecting the solution: the object that has the minimal complexity from among those not discarded, will be reported as the solution to the simplification problem.

Generalization and learning: if the simplification that was applied is significantly different than the source analog it has been derived from, it will be added to the database of known simplifications. Also, if a useful 
generalization over the new simplification and the source simplification can be built, it will also be added to the database.

\subsection{Representing and Organising Design Simplifications}

A simplification is represented by a binary relation (called a simpler-than relation) that connects two objects, a simpler one and a more complicated one (Figure 3). A simpler-then relation has two attributes: an explanation of the simplification it represents and a description of the aspects of the two objects that are relevant to the simplification.

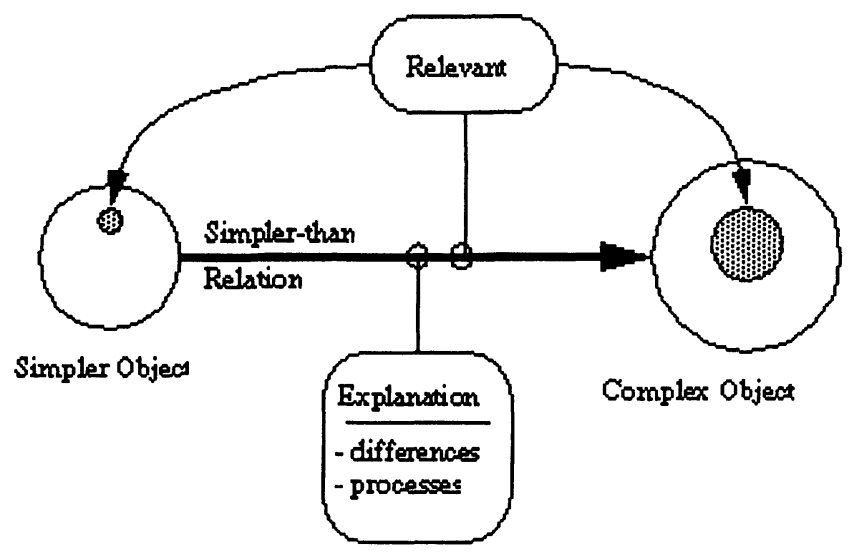

Figure 3. The structure of simplification

The explanation is used for two purposes: it is the basis for determining which elements of the objects involved in the simplification are relevant; it can be used to build abstractions over simplifications, thus organizing simplifications into hierarchies. Hierarchies are useful for the analogical transfer of simplification knowledge, as well as for generating general simplification rules and/or principles.

The 'relevant elements' are used to focus the processing in all phases of analogical reasoning process on only those portions of objects that are involved in some simplification.

The explanation of a simplification can be given by specifying the difference between the two objects involved in the simplification, or by specifying the process by which the simplification was achieved.

Specifying the difference is needed when the fact that an object is simpler than another one was "discovered", but no simplification process is known. How the difference can be specified depends on the ontology used for representing the objects. For example, if the representation uses an objects, components, relations and attributes ontology, the difference can be represented by two sets: a set of elements (components, relations and 
attributes) that are part of the more complicated object, but not part of the simpler one (elements removed), and a set of elements that are part of the simpler object, but not of the more complicated one (elements added).

When the simplification process is known, its description can be added to the simpler relation as an explanation. The process will be represented as a sequence of transformations. Each transformation involves two objects, the transformation operation applied, and the precondition which had to be satisfied in order for the operator to be applicable.

Elements relevant to a simplification (relevant elements) are elements of the designs involved in the simplification that play some role in discovering or explaining the simplification. For example, elements explicitly referred to in the explanation of a simplification are relevant. Relevant elements are useful because they allow the building of abstractions of the objects involved in simplifications. These abstractions will only contain relevant portions of the objects. In addition, the relevant elements can be used as a basis for building indexing schemes over the set of objects involved in known simplifications.

The set of relevant elements corresponding to a given simplification can be computed automatically from the two objects involved in the simplification and the explanation of the simplification. We call the process of finding the relevant elements relevance calculation. Relevant elements can be computed at the time the simplification is created.

The relevance calculation can be decomposed into two phases: a) collecting the elements that are not absolutely irrelevant (with respect to the explanation) and b) propagating relevance along relations in the objects.

Elements not absolutely irrelevant (Levy 1994) with respect to an explanation are elements that are explicitly mentioned in the explanation of the simplification. They may occur in the description of differences, in the case that the explanation is given in terms of differences, or in partial descriptions of objects, specifications of preconditions and arguments of operators, if the explanation is given as a process. These elements are said to be not absolutely irrelevant because, while mentioned in the explanation, they may not be absolutely needed. However there may not be any basis for discarding them as irrelevant.

Note that not absolutely irrelevant elements are elements explicitly present in the explanation of a simplification. These elements may be related to other elements in the design which were not explicitly present in the explanation, but which may bear some relevance to the simplification. This could happen for instance when the explanation of the simplification is given by the difference between the two objects involved. In this case only the removed and added elements are specified, without any reference to relations between them. We conclude that relevance may also need to be propagated 
inside the more complex objects involved in the simplification, along the decompositions, relations and attributes. This propagation can be done either downwards (i.e., from component to subcomponents, from relations to arguments, or components to attributes), or upwards (i.e., from subcomponents to their "parent" component, from components to relations they are arguments of, and from components to relations of which they are attributes).

\subsection{The analogical Reasoning Process}

Retrieving is the first phase of the analogical reasoning process. Its purpose is to find a simplification that corresponds to an object "similar" to the object needing simplification.

For retrieval, simplifications are organized first into classes of simplifications corresponding to points of view, and second, by an indexing scheme over the relevant elements of the objects involved in the simplification. Consequently retrieving similar simplifications also works in two stages: a) pruning, i.e., restricting the search to only the class of simplification with the same point of view as the one specified in the simplification problem, and b) indexing, i.e., search using the indexing schemes. The first stage is implemented by marking each object involved in some simplification that has the corresponding point of view.

The result of retrieving is a set of 'candidate' analogs. Each candidate analog consists of a set of match hypotheses on which its selection as a candidate was based. Each match hypothesis has associated with it a score computed during the retrieval process.

The candidate analog with the highest score is selected to be used in the next phase of the analogical reasoning process. In the rest of this subsection we will assume that a candidate analog has already been selected. As usual, we will refer to the selected candidate analog as the "source" and to the simplification problem as the "target".

Mapping is the second phase of the analogical reasoning process. It builds maximal sets of consistent correspondences (matches) between relevant elements of the source and elements in the target, called global mappings (or gmaps, as they are called in the Structure Mapping Engine, SME, literature). We use a modified version of SME for mapping (Falkenheiner et al., 1993).

SME takes as input two descriptions, one of the source and one of the target, and produces as output a set of gmaps of the source onto the target. Each gmap contains a maximal set of matches. Here 'maximal' means that adding any match to it would violate the consistency of the gmap. SME also 
attaches to each gmap a structural evaluation score which provides an indication of the quality of the mapping.

In our approach to solving the simplification problem by analogical reasoning, the retrieving process associates with each candidate source analog a set of correspondences between relevant elements of the source and elements in the target. Each of these correspondences has assigned to it a score that is an indication of the quality of that correspondence. Our SME implementation uses these correspondences as initial match hypotheses

The mapping process produces a set of global mappings which can be the basis for different simplifications that are likely to be applicable to the given simplification problem. Our purpose is to select the best of these global mappings to increase the chances of generating a simplification in the target. For evaluating global mappings we use a structural evaluation method that assigns higher scores to deeper structures.

At the end of the mapping phase the global mapping with the highest evaluation score will be selected for further consideration in the analogical reasoning process.

Transferring Simplification Knowledge is the next phase. Once a global mapping has been selected as the best candidate for analogical transfer, it will be used to compute candidate simplifications. A candidate simplification is a simplification in the source which can be hypothesized to be applicable in the target as a result of the correspondences of the global mapping.

A candidate simplification is computed by finding elements in the source which are consistent with the global mapping's correspondences, but are not in fact included in them. We will call these elements unbound elements. Unbound elements are searched for in the set of relevant elements of the source since those are the only ones that play some role in that simplification.

Once the unbound elements are found the existence of corresponding elements in the target can be hypothesized. Building these hypotheses is performed by the simplification knowledge transfer process.

How exactly the simplification knowledge will be transferred depends on whether the explanation for the simplification is given by a difference or by a simplification process.

Evaluating the Result of the Simplification is the final phase. Each of the simplified targets resulting from transferring the simplification knowledge and adapting the result has to be evaluated with respect to: a) the requirements on the object, b) the constraints of the simplification and c) the complexity.

After the best simpler object was selected the corresponding simplification can be generated. The target and the new object will be 
respectively the 'more complicated' and 'simpler' objects involved in the simplification. The explanation will be computed as the difference between the two objects, and the relevance calculation will be applied. A design simplification system (Figure 4) using goal-directed analogical reasoning was implemented in CLIPS. It consists of a database of known simplifications, and interface, data management, simplifier, and a simplification abstraction modules.

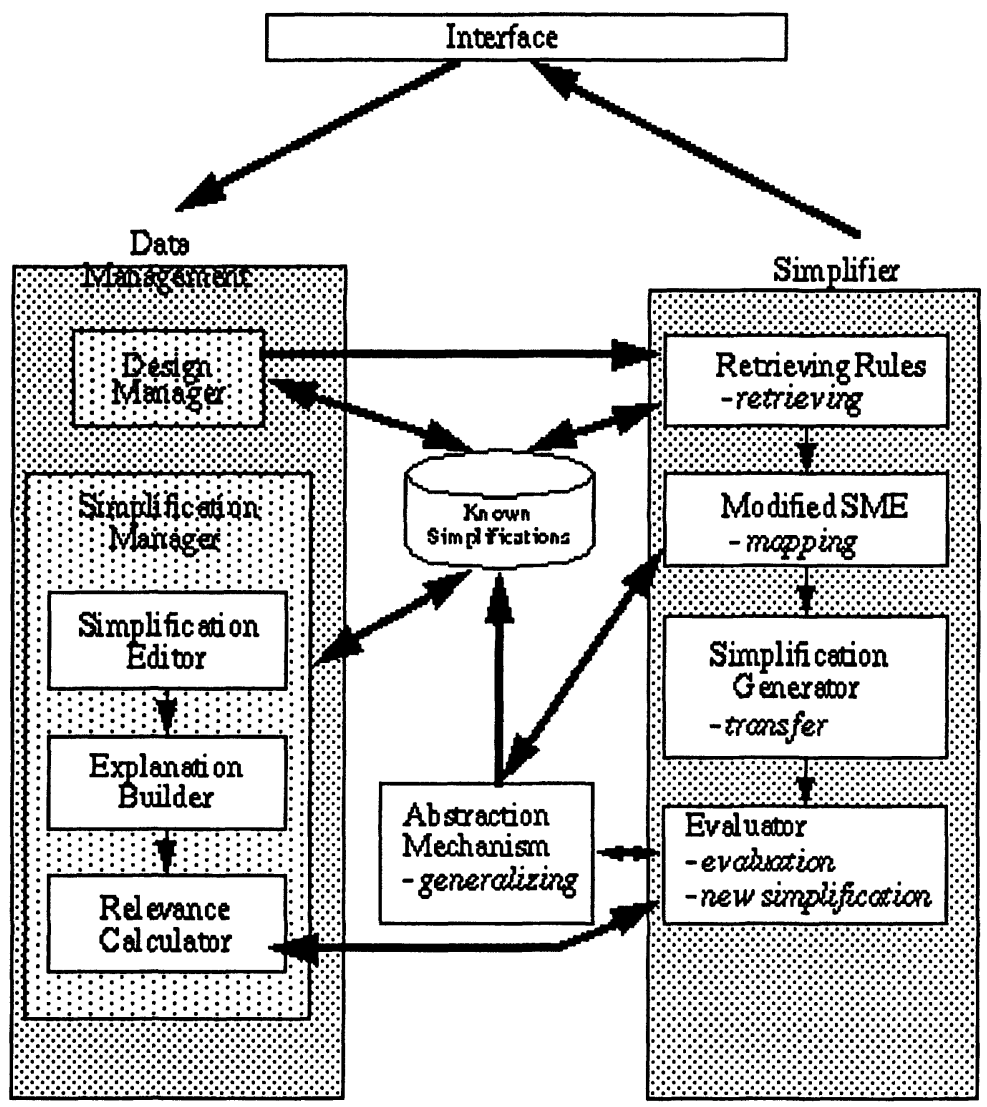

Figure 4. Architecture of the simplification system

\section{ANALYZING THE EFFECT OF USING RELEVANCE}

Applying the relevance of object elements (e.g., sub-expressions of arithmetic expressions, or components of designs) to simplifications throughout the analogical reasoning process is an important contribution. It 
is how the reasoning takes into account the problem solving goal (i.e., simplification), producing goal-directed analogical reasoning.

To demonstrate that using relevance to guide the analogical reasoning process improves system performance, we had to measure its effect. For this we needed: to hypothesize which phases of the analogical reasoning process could be affected by the use of relevance; to define a measure of performance for each of those phases; and to design and perform experiments to collect statistics about the performance measures.

As it is the practice in analyzing algorithm complexity we propose that the time performance of a process should be measured by the number of specific operations performed. Unfortunately there is no unique specific operation that can be counted to measure the system's overall performance. Hence we chose to identify specific operations for each of the phases of the analogical reasoning process that are affected by the use of relevance.

In addition to measuring the performance effects of using relevance, we also studied the effect of different kinds of relevance propagation methods (e.g., downward propagation, limited propagation, no propagation) on the operation of the system. Note that the current implementation does not support upward propagation.

To measure the effect of using relevance on system operation, we hypothesized that the phases affected were: a) the retrieval of source analogs, and b) the mapping of the source analog retrieved onto the target. For both phases we defined a measure of performance in terms of the number of a match hypotheses created for the retrieving phase, and the number of global mappings (called gmaps) generated for the mapping phase.

For the experiments, the system was loaded with a simplification database consisting of simplifications of arithmetic expressions. It contained simplifications explained by difference, as well as by process description.

In one experiment we turned off the relevance checking in the system. Then, for each of the versions of the simplification database (corresponding to different numbers of simplifications) we presented the system with all those examples used in the first set of experiments which produced correct simplifications. We repeated the same set of experiments with the relevance checking turned on.

We also wanted to study whether considering relevant elements to be only the ones explicitly referred to in the explanation of simplifications would make a difference in the performance of the system. For this purpose, we regenerated the databases used for our experiments such that the "relevance propagation" is limited only to the elements explicitly referred to. We then repeated the experiments described above.

The results of the experiments for measuring the effect of using relevance are given in Table 1 . The three main columns in the table 
correspond to performing the experiments without taking into account the relevance, with the relevance fully propagated down, and with the relevance only propagated down one level in the structure of the design matched, respectively. In all the cases the system produced the expected simplification.

Table 1. Summary of experimental results for measuring the effects of using relevance

\begin{tabular}{|c|c|c|c|c|c|c|}
\hline & \multicolumn{2}{|c|}{ Relevance OFF } & \multicolumn{2}{c|}{ Relevance ON } & \multicolumn{2}{|c|}{ One-Level Relevance } \\
\hline $\begin{array}{c}\text { Database } \\
\text { Size }\end{array}$ & $\begin{array}{c}\text { Match } \\
\text { Hypotheses } \\
\text { Generate }\end{array}$ & $\begin{array}{c}\text { Gmaps } \\
\text { Generated }\end{array}$ & $\begin{array}{c}\text { Match } \\
\text { Hypotheses } \\
\text { Generated }\end{array}$ & $\begin{array}{c}\text { Gmaps } \\
\text { Generated }\end{array}$ & $\begin{array}{c}\text { Match } \\
\text { Hypotheses } \\
\text { Generated }\end{array}$ & $\begin{array}{c}\text { Gmaps } \\
\text { Generated }\end{array}$ \\
\hline 18 & 18 & 7 & 11 & 4 & 14 & 6 \\
\hline 40 & 37 & 13 & 27 & 9 & 32 & 11 \\
\hline 50 & 49 & 17 & 36 & 12 & 41 & 17 \\
\hline 75 & 65 & 24 & 41 & 15 & 52 & 21 \\
\hline 85 & 67 & 27 & 56 & 21 & 61 & 33 \\
\hline
\end{tabular}

When taking relevance into account the system generates fewer match hypotheses and, as a consequence, fewer global mappings, leading to a performance improvement. The actual results will depend on the size of the database, the complexity of designs involved in the simplifications and the number of elements in each simplification that are relevant (e.g., for a given simplification it is possible that only one element is relevant, but it is also possible that all the elements of the "simpler" design involved are relevant).

For one-level propagation of relevance the system generates slightly more match hypotheses and gmaps than when using full propagation, but less than when not using propagation at all. This is because one-level propagation restricts the elements that can be matched, but not to the same extent as full propagation. Using full propagation should be the best choice, although this may not always be the case. For simplifications for which the explanation is given by difference, a full propagation may be needed because there is no way of knowing under what conditions the difference is applicable. On the other hand, for simplifications for which the explanation is given by a simplification process description, no propagation should needed, because, ideally, all the relevant elements should be referenced in the process description (in a condition, a transformation, or a state description). As a consequence, the issue of relevance propagation needs further studying. 


\section{RELATED WORK}

We have no knowledge of any ongoing research in the area of "goalbased analogical reasoning for design simplification". However, there is a rich body of research results in model-based analogical reasoning about designs and design optimization.

Model-based analogical reasoning refers to using mental models of the underlying domain in the analogical reasoning process (Gentner 1983). Most of the work on analogical design (Qian \& Gero 1992) (Bhatta \& Goel 1994) (Goel 1997) uses mental models.

Analogical reasoning theory postulates that goals help determine both what gets matched and how the match gets evaluated (Gentner 1993). This idea is incorporated into other analogy research (Holyoak and Thagard 1989) (Forbus and Oblinger 1990). Our approach is related to both. Similar to Forbus \& Oblinger, we propose goal-based filtering. However, in our approach the filtering doesn't only refer to local matches considered, but to designs and design parts based on relevance of their components, attributes and relations. Goal-directed analogical reasoning is not to be mistaken for purpose-directed analogical reasoning (Kedar-Cabelli 1988) which refers to using the purpose of using (the function of) an artifact to guide the analogical reasoning about its structure.

Design simplification, and simplification (as a cognitive activity) in general, is a less researched area. One of the most clear formulations of design simplification principles are Stoll's (1991) design rules for efficient design for manufacture. The only general approach to design simplification we know of is Suh's (1999) "Reduction of the Information Content of a Product". This work gives a formal definition of the information content of a design, which we may interpret as a 'measure of complexity'.

Bashir and Thomson (1999) suggest that the accuracy of estimating the time required by a design project depends on the accuracy of effort estimation. They propose a way to measure complexity of a project in the context of designing it and from the aspect of its function. Boothroyd and Dewhurst (1991) developed a set of DFM/DFA principles for reducing the manufacturing and assembly cost of a product. They view simplification as the reduction of a complexity measure in the context of manufacturing.

\section{CONCLUSIONS \& FUTURE WORK}

The research presented in this paper had two major objectives: a) to define the simplification problem, and b) to propose a way to solve 
simplification problems. In pursuing these objectives this research has produced the following contributions:

- An operational definition of simplification and of a simplification problem.

- A model for solving simplification problems by using analogical reasoning.

- A model-based analogical reasoning approach to design simplification problems.

- A working system that implements the model for solving simplification problems.

- $A$ demonstration that the use of relevance improves the performance of the analogical reasoning mechanism, when generating match hypotheses, retrieving source analogs, and building mappings.

This research opens up new research directions and raises theoretical and practical questions, providing opportunities to extend our research on design simplification. We will perform further experiments with the system using real life design problems.

Currently the system has been tested for structural simplifications, but in our future research we will perform experiments with both behavioral and functional simplification. Adding new application domains will increase the capability of the system to use cross-domain similarities to produce interesting, and hopefully novel simplifications.

The simplification propagation problem (i.e., how simplification in one aspect is propagated to other aspects) raises questions such as: What representation can adequately support the propagation of simplifications? How can the propagation of simplification be performed? What are the possible consequences of simplification propagation and how can those consequences be evaluated and anticipated? In our future research we plan to address these issues.

Finally we expect that due to the use of analogical reasoning, our approach to solving simplification problems may come up with creative simplifications. For example, "importing" a simplification idea from a different domain may suggest a completely novel way of simplifying. We are interested in studying under what conditions our goal-directed analogical reasoning simplification process will be able to produce creative results.

\section{REFERENCES}

Balazs, M.E. (1999). Design Simplification by Analogical Reasoning, Ph.D. Dissertation, WPI.

Bashir, H. A., \& Thomson, V. (1999). A Quantitative Estimation Methodology for Design Projects, In Journal of Engineering Design (submitted). 
Bhatta, S.R., Goel, A., \& Prabhakar, S. (1994). Innovation in analogical design: A modelbased approach, In Gero, J. and Sudweeks, F. (eds.), Proc. Artificial Intelligence in Design '94, Kluwer, pp. 57-74.

Boothroyd, G., \& Dewhurst, P. (1991). Product Design for Manufacture and Assembly, In Corbett, Dooner, Meleka and Pym (eds.) Design For Manufacture, Addison-Wesley, pp. 165-173.

Falkenheiner, B., Forbus K., \& Gentner, D. (1993). The structure-mapping engine: Algorithm and examples, In: Buchanan and Wilkins (eds.) Readings in Knowledge Acquisition and Learning, Morgan Kaufmann Publishers, pp. 695-726.

Forbus, K., \& Oblinger, D. (1990). Making SME greedy and pragmatic. Proc.Cognitive Science Society.

Gentner, D. (1983) "Structure-Mapping: A Theoretical Framework for Analogy", Cognitive Science, 7, 1983, pp. 155-170.

Gentner, D. (1993). "The mechanism of analogical learning", In: Readings in Knowledge Acquisition and Learning, (Eds.) Buchanan \& Wilkins, Morgan Kaufmann Publishers, San Mateo, CA, 1993, pp. 673-694.

Goel, A. (1997). "Design, Analogy and Creativity", IEEE Expert, Vol. 12, No. 3, May/June, 1997, pp.62-70.

Holyoak K.J., \& Thagard, P. (1989). “Analogical mapping by constraint satisfaction", Cognitive Science, 7(2).

Levy, A. Y. (1994). Creating Abstractions Using Relevance Reasoning, In Proceedings of the 12th National Conference on Artificial Intelligence, AAAI'94, Vol. 1, pp. 588-594

Petroski, H. (1996). Invention by Design, Harvard University Press.

Qian, L., \& Gero, J.S. (1992). “A design support systems using analogy”, In: Artificial Intelligence in Design'92, (Ed.) J.S. Gero, Kluwer Academic Publishers, 1992, pp.795813.

Suh, N.P., (1999). "A Theory of Complexity, Periodicity and the Design Axioms", Research in Engineering Design, Vol. 11, No. 2, pp. 116-131. 